\title{
Is it Time to Re-Examine the Physical Exam?
}

\author{
Jeffrey Chi, MD*, Poonam Hosamani, MD¹
}

'Stanford University School of Medicine, Department of Internal Medicine, Division of Hospital Medicine, Stanford, California.

$\mathrm{m}$ I supposed to have such a hard time feeling the kidneys?" "I think I'm doing it wrong," echoed another classmate. The frustration of these first-year students, who were already overwhelmed by the three pages of physical exam techniques that they were responsible for, became increasingly visible as they palpated the abdomens of their standardized patients. Then, they asked the dreaded question: "How often do you do this on real patients?"

When we teach first-year medical students the physical exam, these students are already aware that they have never observed physicians perform these maneuvers in their own medical care. "How come I've never seen my doctor do this before?" is a common question that we are often asked. We as faculty struggle with demonstrating and defending techniques that we hardly ever use given their variable utility in daily clinical practice. However, students are told that they must be familiar with the various "tools" in the repertoire, and they are led to believe that these skills will be a fundamental part of their future practice as physicians. Of course, when they begin their clerkships, the truth is revealed: the currency on the wards revolves around the computer. The experienced and passionate clinicians who may astonish them with the bedside exam are the exception and are hardly the rule.

In this issue of Journal of Hospital Medicine, Bergl et al. ${ }^{1}$ found that when medical students rotated on their internal medicine clerkship, patients were rarely examined during attending rounds and were even examined less often when these rounds were not at the bedside. Although the students themselves consistently incorporated the physical exam into patient assessments and presentations, neither their findings nor those of the residents were ever validated by the attending physician or by others. Notably, the physical exam did not influence clinical decision making as much as one might expect.

These findings should not come as a surprise. The current generation of residents and junior attendings today are more accustomed to emphasizing labs, imaging studies, pathology reports, and other data within the electronic health record $(E H R)$ and with formulating initial plans before having met the patient. ${ }^{2}$ Physicians become uneasy when asked to decide without the reassurance of daily lab results, as if the information in the EHR is highly fundamental to patient care. Caring

*Address for correspondence: Jeffrey Chi, MD, Stanford School of Medicine, Division of Hospital Medicine, 300 Pasteur Drive, HC006, Stanford, CA 94305;

Telephone: (650) 723-8287; Fax: (650) 498-4649; E-mail: jeffrey.chi@stanford.edu

Received: March 9, 2018; Accepted: March 15, 2018

@2018 Society of Hospital Medicine DOI 10.12788/jhm.2991 for the "iPatient" often trumps revisiting and reexamining the real patient. ${ }^{3}$ Medical teams are also bombarded with increasing demands for their attention and time and are pushed to expedite patient discharges while constantly responding to documentation queries in the EHR. Emphasis on patient throughput, quality metrics, and multidisciplinary communication is essential to provide effective patient care but often feels at odds with opportunities for bedside teaching.

Although discussions on these obstacles have increased in recent years, time-motion studies spanning decades and even preceding the duty-hours era have consistently shown that physicians reserve little time for physical examination and direct patient care. ${ }^{4}$ In other words, the challenges in bringing physicians to the bedside might have less to do with environmental barriers than we think.

Much of what we teach about physical diagnosis is imperfect, ${ }^{5}$ and the routine annual exam might well be eliminated given its low yield. ${ }^{6}$ Nevertheless, we cannot discount the importance of the physical exam in fostering the bond between the patient and the healthcare provider, particularly in patients with acute illnesses, and in making the interaction meaningful to the practitioner.

Many of us can easily recall embarrassing examples of obvious physical exam findings that were critical and overlooked with consequences - the missed incarcerated hernia in a patient labeled with gastritis and vomiting, or the patient with chest pain who had to undergo catheterization because the shingles rash was missed. The confidence in normal findings that might save a patient from unnecessary lab tests, imaging, or consultation is often not discussed. The burden is on us to retire maneuvers that have outlived their usefulness and to demonstrate to students the hazards and consequences of poor examination skills. We must also further what we know and understand about the physical exam as Osler, Laennec, and others before us once did. Point-of-care ultrasound is only one example of how innovation can bring trainees to the bedside, excite learners, engage patients, and affect care in a meaningful way while enhancing the nonultrasound-based skills of practitioners.?

It is promising that the students in this study consistently examined their patients each day. As future physicians, they can be very enthusiastic learners eager to apply the physical exam skills they have recently acquired during their early years of training. However, this excitement can taper off if not actively encouraged and reinforced, especially if role models are unintentionally sending the message that the physical exam does not matter or emphasizing exam maneuvers that do not serve a meaningful purpose. New technology will hopefully help us develop novel exam skills. If we can advance what we 
can diagnose at the bedside, students will remain motivated to improve and learn exam skills that truly affect patient-care decisions. After all, one day, they too will serve as role models for the next generation of physicians and hopefully will be the ones taking care of us at the bedside.

Disclosures: The authors declare no conflicts of interest.

\section{References}

1. Bergl PA, Taylor AC, Klumb J, et al. Teaching physical examination to medical students on inpatient medicine reams: A prospective mixed-methods descriptive study. J Hosp Med. 2018;13:399-402.

2. Chi J, Verghese A. Clinical education and the electronic health record: the flipped patient. JAMA. 2010;312(22):2331-2332. DOI: 10.1001/ jama.2014.12820.

2. Verghese A. Culture shock-patient as icon, icon as patient. $N$ Engl J Med. 2008;359(26):2748-2751. DOI: 10.1056/NEJMp0807461

3. Czernik Z, Lin CT. A piece of my mind. Time at the bedside (Computing). JAMA. 2016;315(22):2399-2400. DOI: 10.1001/jama.2016.1722

5. Jauhar S. The demise of the physical exam. N Engl J Med. 2006;354(6):548551. DOI: 10.1056/NEJMp068013

6. Mehrotra A, Prochazka A. Improving value in health care--against the annual physical. N Engl J Med. 2015;373(16):1485-1487. DOI: 10.1056/NEJMp1507485

7. Kugler J. Price and the evolution of the physical examination. JAMA Cardiol. 2018. DOI: 10.1001/jamacardio.2018.0002. [Epub ahead of print] 